\title{
VALORES DE CONSUMO PARA A SUSTENTABILI- DADE: UM ESTUDO COM OS ACADÊMICOS DO CURSO DE CIÊNCIAS CONTÁBEIS DA UNIVERSI- DADE FEDERAL DE SANTA MARIA
}

\author{
CONSUMER VALUES FOR SUSTAINABILITY: A STUDY \\ WITH THE STUDENTS FROM ACCOUNTING OF \\ UNIVERSIDADE FEDERAL DE SANTA MARIA
}

Data de submissão: 20-04-2016 Aceite: 07-07-2016

\author{
Francies Diego Motke \\ Luciana Aparecida Barbieri da Rosa ${ }^{2}$ \\ Letícia Lengler ${ }^{3}$ \\ Jeanne Mainardi ${ }^{4}$ \\ Marcelo Trevisan ${ }^{5}$
}

\section{RESUMO}

Atualmente, é preciso reavaliar o comportamento do consumidor e seus modos de consumo, na tentativa de buscar contribuir para um mundo mais equilibrado - com menos desperdício e impacto ambiental. Nesse sentido, este estudo possui como objetivo analisar o comportamento de consumo sustentável dos acadêmicos do curso de Ciências Contábeis da Universidade Federal de Santa Maria (UFSM). Para tanto, utilizou-se o método survey, com uma amostra caracterizada como não probabilística e por acessibilidade, mediante a aplicação de um questionário que obteve o retorno de 232 respondentes. 0 instrumento de coleta de dados foi adaptado a partir do estudo desenvolvido por Biswas e Roy (2015), que aborda ateoria dos valores de consumo, a qual é composta do valor funcional, valor social, valor condicional, valor ambiental e valor de conhecimento. Os resultados obtidos junto aos acadêmicos da UFSM corroboram o que foi identificado por Biswas e Roy (2015): o valor condicional e o valor ambiental são os fatores que regem o comportamento de consumo sustentável, o que indica que as mudanças na situação econômica dos acadêmicos podem afetar a aquisição de produtos verdes e que eles possuem consciência de que os seus hábitos de consumo afetam o meio ambiente.

Palavras-chave: Sustentabilidade. Consumo. Valores de consumo.

\footnotetext{
1 Possui graduação em Administração pela Universidade Federal de Santa Maria, UFSM, mestrado em Administração pela Universidade Federal de Santa Maria, UFSM. Santa Maria. Rio Grande do Sul. Brasil. E-mail: fdmotke@gmail.com

2 Possui graduação em Administração de Empresas- Bacharelado pela Faculdade de Educação São Luis - FESL e Licenciatura Plena pelo Programa Especial de Graduação de Formação de Professores para a Educação Profissional - PEG / UFSM, especialista em Educação Ambiental pela Universidade Federal de Santa Maria, UFSM, especialista em Gestão Pública pela Universidade Federal de Santa Maria, UFSM, mestrado em Administração de Empresas pela Universidade Federal de Santa Maria, UFSM e doutorado em andamento em Administração pela Universidade Federal de Santa Maria, UFSM. Santa Maria. Rio Grande do Sul. Brasil. E-mail: lucianaaparecidabarbieri@yahoo.com.br

3 Possui graduação em Administração pela Universidade Federal de Santa Maria, UFSM e mestrado em Agronegócios pela Universidade Federal do Rio Grande do Sul, UFRGS. Atualmente é professor assistente da Universidade Federal de Santa Maria. Santa Maria. Rio Grande do Sul. Brasil. E-mail: llenglerworld@yahoo.com

4 Possui graduação em Ciências Contábeis pela Universidade Federal de Santa Maria, UFSM, graduação em Administração pela Universidade Federal de Santa Maria, UFSM e especialização em Gestão Pública pela Universidade Federal do Rio Grande do Sul, UFRGS. Santa Maria. Rio Grande do Sul. Brasil. E-mail: jeannemainardi@gmail.com

5 Possui graduação em Administração pela Universidade Federal de Santa Maria, UFSM, mestrado em Administração pela Universidade Federal de Santa Catarina, UFSC e doutorado em Administração pela Universidade Federal do Rio Grande do Sul, UFRGS. Atualmente é pesquisador colaborador em grupo de pesquisa da Universidade Federal do Rio Grande do Sul, integrante de projeto de pesquisa da Universidade Federal do Rio Grande do Sul e professor adjunto da Universidade Federal de Santa Maria. Santa Maria. Rio Grande do Sul. Brasil. E-mail: marcelotrev@ gmail.com
} 


\section{ABSTRACT}

Lately it is demanded to re-evaluate the consumer behavior and their ways of consumption, since each day it is realized a higher need of awareness about what is being consumed as an attempt to reach and to build a more balanced world - with less waste and environmental impact. In this sense, this study goal is to analyze the sustainable consumption behavior of the students from Accounting of UFSM. Therefore, the survey method was used, with a sample by accessibility characterized as non-probabilistic throughout applying a questionnaire that obtained a return of 442 respondents. The data collect instrument was adapted from the study carried by Biswas and Roy (2015) and it approaches the consumption values theory, composed by functional value, social value, conditional value, environmental value and knowledge value. The results from the UFSM students corroborate with Biswas and Roy (2015) findings: the conditional value followed by the environmental values are the key factors that influence the sustainable consumption behavior, and this indicates that changes in the students economic situation may affect the acquisition for green products and the students are aware of how the environment is affected by their consumption habits.

Keywords: Sustainability. Consumption. Values of consumption.

\section{INTRODUÇÃO}

A globalização e o crescimento econômico levaram a distintas mudanças nas estratégias de produção, refletindo no comportamento de compra dos consumidores. Sabe-se que o consumo desempenha um papel significativo no desenvolvimento humano, tendo se tornado tão dominante economicamente que atingiu proporções preocupantes e impactou intensamente 0 meio ambiente. Em 1992, a Cúpula da Terra criou no Rio de Janeiro a Agenda 21, que é um documento com quatro capítulos dedicados a mudar as caracteríticas de consumo, contibuindo para o desenvolvimento sustentável. No ano de 1998, o Programa das Nações Unidas para o Desenvolvimento (PNUD) enfatizou o conceito de consumo sustentável.

Nesse contexto, entra em cena a temática da educação para a sustentabilidade, que busca influênciar significativamente a consciência ambiental, os estilos de vida diária e o comportamento do consumidor de estudantes. A educação para a sustentabilidade é um processo de sensibilização dos indivíduos, que deve ser iniciado cedo, conscientizando as crianças e permitindo que as transformações sociais em relação à sustentabilidade se tornem mais fáceis. Nesse sentido, a educação exerce um papel fundamental para o desenvolvimento sustentável, pois permite que as pessoas adquiram os conhecimentos, as habilidades e as atitudes necessárias para construir um futuro sustentável (CARS; WEST, 2015).

Várias instituições de ensino têm reconhecido a importância de integrar as questões de sustentabilidade à educação. Ressalta-se que tanto o ensino fundamental quanto o ensino superior possuem a missão de despertar o interesse pela sustentabilidade, procurando desenvolver políticas educacionais que incorpore tal temática nas disciplinas tradicionais, propiciando uma mudança de conceitos e valores, que promovam o consumo consciente.

Segundo Furriela (2001), para conseguir um consumo sustentável, é preciso conscientizar as pessoas da importância de se tornarem consumidores responsáveis e promover um trabalho voltado para a formação de um consumidor-cidadão. Para alcançar esses resultados, o ponto de partida é a sensibilização a respeito da magnitude do problema da degradação dos recursos ambientais, fator que depende de iniciativas na área da educação, já que mudanças nos padrões de consumo envolvem mudanças de comportamento. Considerando esse aspecto, Silva e Gómez (2010) afirmam que a educação surge como transformadora e como direcionadora do indivíduo ao papel de consumidorcidadão, o qual pode assumir uma maior consciência ao incorporar novas responsabilidades. 
Tendo em vista que o consumidor exerce um papel relevante nessa dinâmica de produção versus consumo, o presente estudo buscou analisar o comportamento de consumo sustentável dos acadêmicos do curso de Ciências Contábeis da Universidade Federal de Santa Maria (UFSM). Percebe-se que esse curso em especial, por formar gestores com capacidade e responsabilidades de decidir, exerce um papel relevante no processo da educação para sustentabilidade e consumo consciente. Essa ideia vai ao encontro de que expõem Jacobi, Raufflet e Arruda (2011), ao afirmarem que as universidades precisam reconhecer que possuem um papel muito importante na busca de um futuro global mais sustentável, incorporando esse objetivo na formação dos seus futuros profissionais.

\section{EDUCAÇÃO PARA A SUSTENTABILIDADE E PARA O CONSUMO CONSCIENTE}

As agressões ao ambiente vêm ocorrendo há muitos anos, o que acarreta sérias alterações na natureza. Tais mudanças levam a reflexões e exigem novos posicionamentos dos diversos stakeholders envolvidos no processo de desenvolvimento sustentável. Diante desse quadro, conceitos como o de educação ambiental e consumo consciente têm adquirido maior importância nas agendas dos gestores privados e públicos. Educação ambiental, de acordo com a carta de Belgrado (BARBIERI; SILVA, 2011), é aquela que visa conscientizar os indivíduos sobre o meio ambiente, propiciar a aquisição de valores e motivação (atitudes), proporcionar condições para que os indivíduos e grupos sociais adquiram as habilidades necessárias para proteger e resolver problemas relativos ao ambiente, bem como desenvolver a capacidade de avaliação e de participação, exercitando o senso de responsabilidade e urgência em relação às questões ambientais.

A partir da publicação do relatório "Nosso Futuro Comum", produzido pela Comissão Mundial sobre Meio Ambiente e Desenvolvimento (CMMAD), a expressão desenvolvimento sustentável passou a ser difundida. Esse relatório se refere à educação, em geral, e à educação ambiental, em especial, como formas de alcançar o desenvolvimento sustentável, já que a compreensão dos processos ambientais e de desenvolvimento da maioria das pessoas é baseada em crenças tradicionais e em informações transmitidas pela educação tradicional (CMMAD, 1991).

Barth e Rieckmann (2012) afirmam que a preocupação com a sustentabilidade deve ir além da presença no currículo do ensino superior. Nesse sentido, o desenvolvimento de competências dos docentes é um elemento essencial e pré-requisito para uma mudança de paradigma da sustentabilidade nas Universidades. Os professores devem, assim, ser preparados para lidarem com o assunto, melhorando a aprendizagem prática dos alunos.

Em relação à importância dos professores no processo educacional, Jacobi (2003) afirmar que a educação permite o desenvolvimento de um cidadão com caráter mais propositivo e com capacidade para questionar de forma concreta a falta de iniciativa dos governos e exigir políticas sustentáveis. Os professores desempenham um papel fundamental nesse processo, atuando como mediadores e transmissores de um conhecimento necessário para que os alunos adquiram uma compreensão adequada do meio ambiente, dos problemas, das soluções e da importância da responsabilidade de cada um para construir uma sociedade planetária mais equitativa e ambientalmente sustentável.

O principal legado da educação consiste, assim, na mudança de pensamento, ou seja, na tomada de consciência. Nesse contexto, Silva et al. (2013) entendem que a educação atua como elemento fundamental, pois desencadeia a busca por indagações e respostas mais adequadas, pela conscientização e mudança de comportamento, pela compreensão e disseminação de novas práticas e pelo incentivo ao respeito à vida, por exemplo. 
Roberts (1996) caracteriza os consumidores ecologicamente conscientes como aqueles que compram produtos e serviços que consideram ter impacto positivo (ou menos negativo) no meio ambiente. A esse respeito, Laroche, Bergeron, Barbaro-Forleo (2001) enfatizam que as empresas devem buscar formas de comunicar aos consumidores que a compra de produtos verdes traz benefícios tanto ao bem-estar do consumidor quanto ao meio ambiente.

Ainda conforme Laroche, Bergeron, Barbaro-Forleo (2001), as empresas e os consumidores têm se tornado cada vez mais preocupados com o meio ambiente e estão enfrentando inúmeros desafios em relação ao impacto da produção e do comportamento de consumo. No entanto, Kolmuss e Angyeman (2002) alertam que o aumento do conhecimento dos consumidores sobre os problemas ambientais, embora desperte a consciência e a preocupação desses indivíduos, não necessariamente resulta em mudanças comportamentais. Nesse mesmo sentido, Marjainé et al. (2011) explicitam que as mudanças nas atitudes e nos valores são os requisitos necessários para que possa haver um comportamento diferente, no sentido de buscar um outro rumo para a sociedade, dentro dos preceitos discutidos em prol da sustentabilidade.

\section{VALORES DO CONSUMO RELACIONADO À DECISÃO DE COMPRA DE PRODUTOS VERDES}

O ambiente competitivo fez com que as empresas de modo geral buscassem estratégias que causassem menos impactos advindos do processo de fabricação de seus produtos. Os estudos sobre comportamento do consumidor em relação a produtos verdes começaram a serem realizados a partir da década de 70 , quando variáveis como valores, crenças, conhecimentos, necessidades, motivações, atitudes e demografia passam a ser analisadas como fatores que influenciam a escolha dos consumidores no que diz respeito à compra desses produtos (PEATTIE, 2010).

Apesar de não existir um consenso sobre a definição de produto verde, em função desse termo ser relativamente novo, Michaud e Llerena (2011) entendem que os produtos verdes têm as mesmas funções básicas que os produtos convencionais, porém seu impacto sobre seu ciclo de vida é mitigado

No entanto, somente a partir da década de 90 , os consumidores começaram a se preocupar com o impacto causado pelos produtos consumidos no meio ambiente. Nesse momento, os clientes-chave, com uma visão de consumo verde, começaram a exigir que as empresas buscassem uma atitude pró-ativa em relação ao meio ambeinte e às questões sociais (POLONSKY; ROSENBERGER, 2001).

Nesse contexto, a Agenda 21 aborda a relevância de atentar para o consumo como causador de diferentes impactos ambientais e sociais, conceituando o consumo sustentável como aquele que envolve a escolha de produtos que utilizaram menos recursos naturais em sua produção, que garantiram o emprego decente aos que os produziram, e que serão facilmente reaproveitados ou reciclados. Significa comprar aquilo que é realmente necessário, estendendo a vida útil dos produtos tanto quanto possível. Consumimos de maneira sustentável quando nossas escolhas de compra são conscientes, responsáveis, com a compreensão de que terão consequências ambientais e sociais - positivas ou negativas (MINISTÉRIO DO MEIO AMBIENTE, 2015).

A partir de tais pressões, as organizações inseriram em seus processos tecnologias limpas, além de conscientizar seus consumidores acerca da importância do consumo de produtos verdes para garantir a sustentabilidade das futuras gerações (RA'NAEE; KORDSHULI; BUZANJANI, 2012). Pode-se afirmar, assim, que o consumo sustentável se refere ao padrão de redução do consumo de recursos naturais, à mudança do estilo de vida e ao consumo de produtos verdes para satisfazer as atuais necessidades e as aspirações das gerações futuras. 
Nesse contexto, o esforço para compreender o comportamento do consumo sustentável tornou-se onipresente entre os grupos acadêmicos nos últimos anos, de forma que muitos modelos têm sido desenvolvidos para explicar o comportamento de consumo sustentável ou comportamento de consumo verde (WANG et al., 2013; ZSOKA et al., 2013). Dentre os modelos desenvolvidos, pode ser visualizado na Tabela 1,exposta a seguir, o modelo de Biswas e Roy (2015), que salientam que o consumo e os estilos de vida adaptados aos hábitos e contextos são propensos a mudar de acordo com certos valores pessoais; com a percepção e informação (valor ambiental e conhecimento); com a influência do contexto social sobrea imagem, a aceitação e a identidade do grupo de pares (valores sociais); e com a oportunidade, tal como disponibilidade de alternativas à concorrência de preços (valor condicional).

Tabela 1 - Influência de valores de consumo sobre o comportamento de escolha do consumidor

\begin{tabular}{|c|c|c|}
\hline Tipos de valores & Descrição & Bases teóricas \\
\hline Valor funcional & $\begin{array}{l}\text { É medido pela percepção dos consumidores sobre o de- } \\
\text { sempenho do produto quanto à durabilidade, à perma- } \\
\text { nência, à confiabilidade, ao preço e à qualidade. É avaliado } \\
\text { como o principal condutor de comportamento de escolha } \\
\text { do consumidor em decisão de compra de produtos verdes. }\end{array}$ & $\begin{array}{l}\text { Shethet al. (1991) e } \\
\text { Bei e Simpson (1995) }\end{array}$ \\
\hline Valor social & $\begin{array}{c}\text { É um valor derivado da associação com um ou mais grupos } \\
\text { sociais. No entanto, vários estudos sugerem que as deci- } \\
\text { sões dos consumidores são mais influenciadas por fatores } \\
\text { pessoais, como atitude e traços de personalidade, do que } \\
\text { normas sociais ou pressão. }\end{array}$ & $\begin{array}{l}\text { Shethet al. (1991) e } \\
\text { Bei e Simpson (1995) }\end{array}$ \\
\hline Valor condicional & $\begin{array}{l}\text { Indica a utilidade derivada de uma situação específica. } \\
\text { Pesquisas reconhecem que as mudanças na situação do } \\
\text { consumidor e variáveis adicionais podem afetar a adoção } \\
\text { de produtos verdes. }\end{array}$ & $\begin{array}{l}\text { Saxena e Khandelwal } \\
\text { (2010), Niemeyer } \\
\text { (2010) e Gadenneet } \\
\text { al. (2011) }\end{array}$ \\
\hline Valor ambiental & $\begin{array}{c}\text { Diz respeito às noções relativamente estáveis sobre ques- } \\
\text { tões como limite da população e relação entre meio am- } \\
\text { biente e desenvolvimento. }\end{array}$ & $\begin{array}{l}\text { Kilbourne e Pickett } \\
\text { (2008), Wang et al. } \\
\text { (2013) e Rex e Bau- } \\
\text { mann (2007) }\end{array}$ \\
\hline $\begin{array}{c}\text { Valor de } \\
\text { conhecimento }\end{array}$ & $\begin{array}{l}\text { Refere-se à utilidade percebida para satisfazer a falta de co- } \\
\text { nhecimento e busca de novidades. A falta de informações } \\
\text { sobre produtos verdes, muitas vezes, resulta em uma dife- } \\
\text { rença de comportamento na atitude do consumidor. }\end{array}$ & $\begin{array}{l}\text { Larocheet al. (2001) } \\
\text { e Tanner e Kast } \\
\qquad(2003)\end{array}$ \\
\hline
\end{tabular}

Fonte: elaborada pelos autores com base no estudo de Biswas e Roy (2015).

Em um estudo conduzido na China por Lin e Huang (2012), contudo, foi possível identificar que o fator que mais influencia os consumidores de produtos verdes não é o valor funcional (com relação ao preço e à qualidade), resultado que diverge da conclusão de Biswas e Roy (2015). No estudo indiano, os respondentes que participaram de duas conferências na Índia se mostraram mais suscetíveis ao preço dos produtos, sendo o valor condicional o mais valorizado. Apesar de ambos os estudos procurarem identificar como os valores de consumo são percebidos, não se pode desconsiderar a diferença cultural entre a China e a Índia. Os dois países continuam com a maior população excluída e miserável do planeta e são, hoje, o foco de comparações inesgotáveis a respeito de seus respectivos processos de desenvolvimento econômico. 
Nessa perspectiva, o comportamento de escolha dos consumidores por produtos verdes possui grande importância para os gestores e fornecedores interessados em estimular a procura de produtos verdes, a fim de aumentar sua participação de mercado para produtos ambientalmente sustentáveis. Tendo isso em vista, neste estudo, utilizou-se como base o modelo desenvolvido por Biswas e Roy (2015), composto de 20 questões relativas aos cinco tipos de valores citados anteriormente, para compreender o consumo de produtos verdes.

\section{METODOLOGIA}

Este estudo se caracteriza, quanto aos objetivos, como descritivo, uma vez que pretende descrever os fatos e fenômenos de determinada realidade (TRIVINOS, 1987). Quanto à abordagem, caracteriza-se como quantitativo, método que, de acordo com o Richardson (1999), consiste no emprego da quantificação na coleta e no tratamento das informações por meio de técnicas estatísticas.

Além disso, utilizou-se o método survey, que é um método quantitativo que busca a obtenção de dados ou informações sobre características, ações ou opiniões de determinado grupo de pessoas, indicado como representante de uma população-alvo, por meio de um instrumento de pesquisa, normalmente um questionário (PINSONNEAULT; KRAEMER, 1993). Para analisar qual a percepção e conscientização dos estudantes universitários em relação ao comportamento de consumo sustentável, ou seja, do consumo de produtos verdes, escolheu-se como objeto de estudo os acadêmicos do curso de Ciências Contábeis da UFSM. Como instrumento de coleta de dados, trabalhou-se com um questionário estruturado composto de perguntas fechadas e adaptado do estudo desenvolvido por Biswas e Roy (2015) que teve como objetivo analisar o comportamento de consumo sustentável desses.

O questionário foi dividido em duas partes. A primeira parte era destinada a identificar a frequência das percepções frente ao consumo sustentável, sendo composta de 20questões sobre a teoria dos valores de consumo (constituída pelos valores funcional, social, condicional, ambiental e de conhecimento) e formulada com base em uma escala Likert de cinco pontos, em que (1) significa "discordo totalmente", (2) significa "discordo parcialmente", (3) significa "indiferente", (4) significa "concordo parcialmente" e (5) significa "concordo totalmente". Já a segunda parte buscou caracterizar o perfil dos respondentes.

Os questionários foram aplicados em junho de 2015 de forma presencial, gerando um retorno de 232 respondentes, e a amostra foi caracterizada como não probabilística e por acessibilidade. Posteriormente, os dados foram tabulados em uma planilha eletrônica, por meio do programa Microsoft Excel 2013, e analisados com o auxílio do programa Statistical Package for the Social Sciences (SPSS) 22, que permite realizar análises estatísticas. Os resultados são apresentados em forma de tabelas, analisados individualmente pelos pesquisadores e descritos em forma de texto na seção seguinte.

\section{ANÁLISE E DISCUSSÃO DOS RESULTADOS}

Os resultados desta pesquisa são apresentados na seguinte ordem: caracterização da amostra; análises descritivas das variáveis e dos constructos; e influência do perfil dos respondentes nas variáveis.

\subsection{Caracterização da amostra}

Evidenciou-se que, entre os respondentes, 147 pertencem ao gênero feminino (63,4\%) e 85 são do gênero masculino (36,6\%). Quanto à idade, a maior parcela dos estudantes possui 
entre 21 e 24 anos ( $n=86,40 \%), 35,8 \%$ têm até 20 anos ( $n=77$ ), e $24,2 \%$ possuem 25 anos ou mais $(n=52)$. Em relação ao turno do curso dos estudantes de Ciências Contábeis, $56 \%$ são discentes do curso noturno ( $n=130)$, e $44 \%$ pertencem ao curso diurno ( $n=102)$.

A Tabela 2, a seguir, apresenta os dados gerais dos estudantes que participaram desta pesquisa.

Tabela 2 - Dados gerais dos respondentes

\begin{tabular}{|c|c|}
\hline Gênero & Semestre \\
\hline Masculino $(36,6 \%)$ & 1 o semestre $(12,1 \%)$ \\
\hline Feminino $(63,4 \%)$ & 20 semestre $(9,5 \%)$ \\
\hline Idade & 3ㅇs semestre $(6,5 \%)$ \\
\hline Até 20 anos $(35,8 \%)$ & 40 semestre $(12,1 \%)$ \\
\hline De 21 a 24 anos (40\%) & 5o semestre $(13,8 \%)$ \\
\hline Mais de 24 anos $(24,2 \%)$ & 60 semestre $(12,5 \%)$ \\
\hline Turno & 70 semestre $(12,5 \%)$ \\
\hline Noturno (56\%) & 8 o semestre $(12,1 \%)$ \\
\hline Diurno (44\%) & 9o semestre $(9,1 \%)$ \\
\hline
\end{tabular}

Fonte: elaborada pelos autores.

A partir da análise da Tabela 2, pode-se concluir que a maioria dos estudantes participantes da pesquisa é do sexo feminino (63,4\%), possui entre 21 e 24 anos (40\%), frequenta o curso noturno de Ciências Contábeis (56\%) e está no 5o semestre (13,8\%).

\subsection{Análises descritivas das variáveis e dos constructos}

A seguir, apresentam-se os resultados da pesquisa com base nas análises descritivas das variáveis e dos constructos. Na Tabela 3, é possível visualizar a média e o desvio padrão de cada variável para posterior análise.

Tabela 3 - Média e desvio padrão das variáveis

\begin{tabular}{|c|c|c|}
\hline Valor funcional & Média & $\sigma$ \\
\hline FUNC1 - Os produtos sustentáveis são bons produtos pelo preço cobrado. & 2,987 & 1,0042 \\
\hline FUNC2 - Os produtos sustentáveis são baratos pelos atributos que oferecem. & 2,728 & 1,0809 \\
\hline FUNC3 - Os produtos sustentáveis possuem um padrão de qualidade esperado. & 3,616 & 0,8799 \\
\hline FUNC4 - Os produtos sustentáveis são feitos de substâncias não perigosas. & 3,552 & 1,1308 \\
\hline Valor social & Média & $\sigma$ \\
\hline SOC1 - Comprar produtos sustentáveis irá me ajudar a obter aceitação social. & 2,457 & 0,9882 \\
\hline $\begin{array}{l}\text { SOC2 - Comprar produtos sustentáveis trará uma impressão positiva para os meus gru- } \\
\text { pos sociais (pessoas de relações mais próximas). }\end{array}$ & 2,978 & 0,9866 \\
\hline $\begin{array}{l}\text { SOC3 - Eu compraria produtos sustentáveis por sugestão dos grupos sociais dos quais } \\
\text { eu participo, que preferem comprar estes produtos. }\end{array}$ & 3,242 & 1,1237 \\
\hline SOC4 - A compra de produtos sustentáveis melhoraria a forma como sou percebido. & 3,121 & 0,9273 \\
\hline
\end{tabular}




\begin{tabular}{|c|c|c|}
\hline Valor condicional & Média & $\sigma$ \\
\hline $\begin{array}{l}\text { COND1 - Compraria produtos sustentáveis ao invés de produtos convencionais se hou- } \\
\text { vessem descontos ou promoções. }\end{array}$ & 4,474 & 0,7725 \\
\hline $\begin{array}{l}\text { COND2 - Compraria produtos sustentáveis ao invés de produtos convencionais se hou- } \\
\text { vesse subsídio para os produtos sustentáveis. }\end{array}$ & 3,649 & 0,9292 \\
\hline $\begin{array}{l}\text { COND3 - Compraria produtos sustentáveis ao invés de produtos convencionais quando } \\
\text { aqueles estivessem disponíveis, ou seja, pudessem ser adquiridos facilmente. }\end{array}$ & 3,991 & 0,9551 \\
\hline $\begin{array}{l}\text { COND4 - Compraria produtos sustentáveis ao invés de produtos convencionais para } \\
\text { evitar piora das condições ambientais. }\end{array}$ & 4,160 & 0,9396 \\
\hline Valor ambiental & Média & $\sigma$ \\
\hline $\begin{array}{l}\text { AMB1 - A poluição ambiental e a escassez de recursos naturais têm ameaçado as futu- } \\
\text { ras gerações. }\end{array}$ & 4,810 & 0,5809 \\
\hline AMB2 - O equilíbrio da natureza é muito delicado e facilmente perturbado. & 4,478 & 0,8268 \\
\hline AMB3 - Nós estamos nos aproximando do limite sustentável da Terra. & 3,762 & 1,1977 \\
\hline $\begin{array}{l}\text { AMB4 - Quando eu tenho escolha entre dois produtos iguais, eu compro o menos pre- } \\
\text { judicial para o ambiente e para a sociedade. }\end{array}$ & 3,621 & 1,0824 \\
\hline $\begin{array}{l}\text { AMB5 - Dou preferência para o consumo de produtos de empresas que investem na } \\
\text { preservação do meio ambiente. }\end{array}$ & 3,246 & 1,0257 \\
\hline Valor de conhecimento & Média & $\sigma$ \\
\hline $\begin{array}{l}\text { CONH1 - Eu prefiro verificar os rótulos e as certificações que informam se o produto é } \\
\text { sustentável (ou ecológico, ou orgânico) antes de comprá-lo. }\end{array}$ & 2,780 & 1,0564 \\
\hline $\begin{array}{l}\text { CONH2 - Prefiro receber informações substanciais sobre os produtos sustentáveis antes } \\
\text { de comprá-los. }\end{array}$ & 3,707 & 0,9980 \\
\hline $\begin{array}{l}\text { CONH3 - Busco informações a respeito dos insumos, processos e impactos relativos aos } \\
\text { produtos antes de comprá-los. }\end{array}$ & 2,599 & 1,0806 \\
\hline
\end{tabular}

Como pode ser observado na Tabela 3, as médias estão situadas entre os valores de 2,457 (menor média) e 4,810 (maior média). Observa-se, ainda, que as variáveis com médias mais altas, em geral, apresentam um desvio padrão mais baixo que as demais, o que demonstra certa conformidade de opiniões para estas variáveis.

A variável que apresentou a maior média $(4,810)$ foi AMB1, "A poluição ambiental e a escassez de recursos naturais têm ameaçado as futuras gerações", do constructo valor ambiental, o que indica que os estudantes de Ciências Contábeis entrevistados estão preocupados com assuntos como poluição e escassez de recursos, bem como com o impacto que isso pode causar no futuro. A vaiável AMB2, "O equilíbrio da natureza é muito delicado e facilmente perturbado", do constructo valor ambiental, também apresentou uma média alta $(4,478)$, revelando que os respondentes têm clareza do impacto que o homem pode causar na natureza com atitudes que vão contra os princípios sustentáveis.

Outras variáveis que apresentaram médias altas foram COND1, "Compraria produtos sustentáveis ao invés de produtos convencionais se houvessem descontos ou promoções", com média de 4,474, e COND4, "Compraria produtos sustentáveis ao invés de produtos convencionais para evitar piora das condições ambientais", com média de 4,160, ambas do constructo valor condicional. A primeira revela que estes estudantes ainda consideram os produtos sustentáveis muito caros para serem adquiridos e que o simples fato do produto oferecer algum benefício ambiental e/ou social pode não ser suficiente para a compra. Já a segunda variável indica que, apesar de o preço ser um fator importante na escolha de um produto, conforme revelado pela 
variável COND1, os respondentes mostram-se dispostos a investir em produtos sustentáveis à medida que estes produtos possam trazer benefícios às atuais condições ambientais. Nota-se, até aqui, que as quatro variáveis que apresentaram maiores médias pertencem aos constructos valor ambiental e valor condicional, o que pode revelar que estes estudantes, embora já tenham adquirido certa consciência ambiental, ainda necessitam de algumas condições/intervenções para que tenham um consumo mais consciente.

Ressalta-se, ainda, que algumas variáveis apresentaram médias mais baixas em comparação com as demais. A variável SOC1, “Comprar produtos sustentáveis irá me ajudar a obter aceitação social", do constructo valor social, obteve a menor média $(2,457)$, indicando que a aquisição de produtos sustentáveis não traz impacto positivo na relação com seu grupo social. A variável CONH3,"Busco informações a respeito dos insumos, processos e impactos relativos aos produtos antes de comprá-los", do constructo valor de conhecimento, também apresentou uma média baixa $(2,599)$, o que demonstra que os respondentes não costumam fazer uma análise mais aprofundada sobre a sustentabilidade do produto antes de adquiri-lo.

Outras variáveis que apresentaram médias mais baixas que as demais foram a variável FUNC2 "Os produtos sustentáveis são baratos pelos atributos que oferecem", do constructo valor funcional, com média de 2,728, e a variável CONH1, "Eu prefiro verificar os rótulos e as certificações que informam se o produto é sustentável (ou ecológico, ou orgânico) antes de comprá-lo", do constructo valor de conhecimento, com média de 2,780. A primeira indica que o preço é um fator limitador para a compra de mais produtos sustentáveis, e a segunda indica que os estudantes entrevistados não costumam analisar os rótulos dos produtos para verificar se são ou não sustentáveis.

A seguir, na Tabela 4, apresenta-se a média de cada constructo pertencente ao modelo adotado para posterior análise.

Tabela 4 - Média dos constructos

\begin{tabular}{c|c}
\hline Constructos & Média \\
\hline Valor condicional & 4,068 \\
\hline Valor ambiental & 3,983 \\
\hline Valor funcional & 3,221 \\
\hline Valor de conhecimento & 3,029 \\
\hline Valor social & 2,949 \\
\hline
\end{tabular}

Fonte: elaborada pelos autores.

Observa-se, na Tabela 4, que os constructos com as médias mais elevadas dizem respeito ao valor condicional, com média de 4,068, e ao valor ambiental, com média de 3,983. O constructo valor condicional indica que algumas mudanças em variáveis situacionais podem contribuir para uma maior adoção de produtos verdes entre os respondentes. Já o constructo valor ambiental indica que grande parte dos participantes da pesquisa tem a consciência de que os hábitos de consumo podem afetar o meio ambiente. Segundo Rex e Baumann (2007), as emoções que o indivíduo exibe para proteção e melhoria do meio ambiente irão desencadear decisões de compras verdes.

Em relação às médias mais baixas dos constructos analisados, o constructo valor social, com média de 2,949, demonstra que as pressões sociais podem não ser suficientes para a prática de consumo sustentável entre os participantes. A esse respeito, Biswas e Roy (2015) relatam que vários estudos sugerem que as decisões dos consumidores são mais influenciadas por fatores pessoais, como atitude e traços de personalidade, do que normas sociais ou pressões. Já o constructo valor de conhecimento, com média de 3,029, aponta que a busca por informações a respeito dos produtos que são adquiridos pelos respondentes não é uma prática frequente. 


\subsection{Influência do perfil dos respondentes nas variáveis}

Os procedimentos estatísticos adotados nesta etapa tiveram como objetivo calcular a média e o desvio padrão das variáveis e, após, realizar testes estatísticos de diferença de médias (teste T e teste ANOVA), para verificar se as diferenças foram significativamente representativas, ou seja, se apresentaram significância inferior a 0,05. A seguir, a Tabela 5 apresenta as variáveis que sofrem influência do gênero dos respondentes.

Tabela 5 - Influência do gênero nas variáveis

\begin{tabular}{c|c|c|c|c|c}
\hline Variáveis & Gênero & Média & $\boldsymbol{\sigma}$ & T & Sig \\
\hline \multirow{2}{*}{$\begin{array}{c}\text { FUNC1 - Os produtos sustentáveis são bons } \\
\text { produtos pelo preço cobrado. }\end{array}$} & Masculino & 2,788 & 1,0701 & & \multirow{2}{*}{0,026} \\
\cline { 2 - 4 } & Feminino & 3,102 & 0,9489 & & \\
\hline \multirow{2}{*}{$\begin{array}{c}\text { FUNC3 - Os produtos sustentáveis possuem } \\
\text { um padrão de qualidade esperado. }\end{array}$} & Masculino & 3,318 & 1,0025 & & \multirow{2}{*}{0,000} \\
\cline { 2 - 4 } & Feminino & 3,789 & 0,7515 & & \\
\hline
\end{tabular}

Fonte: elaborada pelos autores.

Observa-se na Tabela 5 que, para as duas variáveis que obtiveram diferenças significativamente representativas nas médias, os respondentes do gênero feminino apresentaram as maiores médias. Nas variáveis FUNC1, "Os produtos sustentáveis são bons produtos pelo preço cobrado", e FUNC3, "Os produtos sustentáveis possuem um padrão de qualidade esperado", observa-se que, entre os respondentes, os estudantes do gênero feminino consideram a relação qualidade-preço dos produtos sustentáveis mais satisfatória que os estudantes do sexo masculino (médias de 3,082 e 3,696, respectivamente).

A Tabela 6 , a seguir, demonstra as variáveis que apresentaram diferenças significativamente representativas quando analisadas sob a influência da idade.

Tabela 6 - Influência da idade nas variáveis

\begin{tabular}{|c|c|c|c|c|c|}
\hline Variável & Idade & Média & $\sigma$ & $\mathbf{F}$ & Sig \\
\hline \multirow{3}{*}{$\begin{array}{l}\text { AMB4 - Quando eu tenho escolha entre dois } \\
\text { produtos iguais, eu compro o menos prejudi- } \\
\text { cial para o ambiente e para a sociedade. }\end{array}$} & Até 20 anos & 3,753 & 1,1021 & \multirow{3}{*}{3,983} & \multirow{3}{*}{0,020} \\
\hline & De 21 a 24 anos & 3,360 & 1,0728 & & \\
\hline & Mais de 24 anos & 3,827 & 1,0796 & & \\
\hline \multirow{3}{*}{$\begin{array}{l}\text { CONH1 - Eu prefiro verificar os rótulos e cer- } \\
\text { tificações que informam se o produto é sus- } \\
\text { tentável (ou ecológico, ou orgânico) antes de } \\
\text { comprá-lo. }\end{array}$} & Até 20 anos & 2,649 & 1,0484 & \multirow{3}{*}{3,117} & \multirow{3}{*}{0,046} \\
\hline & De 21 a 24 anos & 2,721 & 1,0250 & & \\
\hline & Mais de 24 anos & 3,096 & 1,0712 & & \\
\hline \multirow{3}{*}{$\begin{array}{c}\text { CONH3 - Busco informações a respeito dos } \\
\text { insumos, processos e impactos relativos aos } \\
\text { produtos antes de comprá-los. }\end{array}$} & Até 20 anos & 2,610 & 1,0407 & \multirow{3}{*}{3,133} & \multirow{3}{*}{0,046} \\
\hline & De 21 a 24 anos & 2,430 & 1,0237 & & \\
\hline & Mais de 24 anos & 2,904 & 1,2088 & & \\
\hline
\end{tabular}

Fonte: elaborada pelos autores.

Percebe-se na Tabela 6 que, para todas as variáveis que apresentaram diferenças significativas nas médias (AMB4, "Quando eu tenho escolha entre dois produtos iguais, eu compro o menos prejudicial para o ambiente e para a sociedade", $\mathrm{CONH1}$, "Eu prefiro verificar os rótulos e certificações que informam se o produto é sustentável (ou ecológico, ou orgânico) antes de comprá-lo", e CONH3, "Busco informações a respeito dos insumos, processos e impactos relativos 
aos produtos antes de comprá-los"), as médias maiores dizem respeito ao grupo de maior idade $(3,827,3,096$ e 2,904, respectivamente). Tais resultados revelam que os estudantes de Ciências Contábeis situados em uma faixa etária mais elevada são os que mais têm preferência pela compra de produtos sustentáveis e são os que mais buscam informações acerca da sustentabilidade dos produtos que consomem.

A seguir, na Tabela 7, pode-se visualizar a variável que apresentou diferença significativa nas médias quando analisada em relação ao turno do curso frequentado pelo respondente.

Tabela 7 - Influência do turno nas variáveis

\begin{tabular}{c|c|c|c|c|c}
\hline Variável & Turno & Média & $\boldsymbol{\sigma}$ & $\mathbf{T}$ & Sig \\
\hline \multirow{2}{*}{$\begin{array}{c}\text { Soc4 - A compra de produtos sustentáveis } \\
\text { melhoraria a forma como sou percebido. }\end{array}$} & Noturno & 2,992 & 0,9685 & \multirow{2}{*}{0,405} & \multirow{2}{*}{0,017} \\
\cline { 1 - 3 } & Diurno & 3,284 & 0,8487 & & \\
\hline Fonte: elaborada pelos autores.
\end{tabular}

Com base nesses resultados, percebe-se que os estudantes do curso de Ciências Contábeis noturno que responderam a pesquisa são os que mais confiam que a melhorariam a forma como são percebidos se comprassem produtos sustentáveis, conforme a média 3,284 da variável SOC4,"A compra de produtos sustentáveis melhoraria a forma como sou percebido para este grupo", em comparação à média de 2,992 para os estudantes do curso diurno de Ciências Contábeis.

A Tabela 8, a seguir, apresenta as variáveis que demonstraram diferenças significativas nas médias quando analisado o semestre do respondente.

Tabela 8 - Influência do semestre nas variáveis

\begin{tabular}{|c|c|c|c|c|c|}
\hline Variável & Idade & Média & $\sigma$ & $F$ & Sig \\
\hline \multirow{9}{*}{$\begin{array}{l}\text { SOC1 - Comprar produtos sustentáveis irá me ajudar a } \\
\text { obter aceitação social. }\end{array}$} & 10 semestre & 2,357 & 0,8262 & \multirow{9}{*}{2,456} & \multirow{9}{*}{0,014} \\
\hline & 20 semestre & 2,045 & 1,0455 & & \\
\hline & 30 semestre & 2,267 & 0,9612 & & \\
\hline & 40 semestre & 2,321 & 0,9833 & & \\
\hline & 50 semestre & 2,781 & 0,9413 & & \\
\hline & 60 semestre & 2,241 & 0,9124 & & \\
\hline & 70 semestre & 2,793 & 1,0481 & & \\
\hline & 8 semestre & 2,821 & 0,9833 & & \\
\hline & 9o semestre & 2,190 & 0,9808 & & \\
\hline \multirow{9}{*}{$\begin{array}{l}\text { COND2 - Compraria produtos sustentáveis ao invés pro- } \\
\text { dutos convencionais se houvesse subsídio para os produ- } \\
\text { tos sustentáveis. }\end{array}$} & 1ㅇ semestre & 3,286 & 0,8100 & \multirow{9}{*}{2,168} & \multirow{9}{*}{0,031} \\
\hline & 20 semestre & 3,091 & 0,5264 & & \\
\hline & 3o semestre & 3,733 & 1,1629 & & \\
\hline & 40 semestre & 3,679 & 0,9449 & & \\
\hline & 5o semestre & 3,742 & 1,0945 & & \\
\hline & 60 semestre & 3,931 & 0,8836 & & \\
\hline & 70 semestre & 3,759 & 0,8724 & & \\
\hline & 80 semestre & 3,786 & 0,7868 & & \\
\hline & 9o semestre & 3,762 & 1,0443 & & \\
\hline
\end{tabular}




\begin{tabular}{|c|c|c|c|c|c|}
\hline Variável & Idade & Média & $\sigma$ & $F$ & Sig \\
\hline \multirow{9}{*}{$\begin{array}{l}\text { AMB3 - Nós estamos nos aproximando do limite susten- } \\
\text { tável da Terra. }\end{array}$} & 10 semestre & 4,000 & 0,7201 & \multirow{9}{*}{2,117} & \multirow{9}{*}{0,035} \\
\hline & 20 semestre & 3,048 & 1,0713 & & \\
\hline & 30 semestre & 3,400 & 1,4541 & & \\
\hline & 40 semestre & 3,464 & 1,3189 & & \\
\hline & 5o semestre & 3,844 & 0,9541 & & \\
\hline & 60 semestre & 3,897 & 1,3187 & & \\
\hline & 70 semestre & 4,000 & 1,3093 & & \\
\hline & 80 semestre & 4,143 & 1,0079 & & \\
\hline & 9o semestre & 3,667 & 1,4259 & & \\
\hline
\end{tabular}

Fonte: elaborada pelos autores.

De acordo com a Tabela 8, nota-se que, para todas as variáveis que apresentaram diferenças significativas nas médias (SOC1, "Comprar produtos sustentáveis irá me ajudar a obter aceitação social", COND2, "Compraria produtos sustentáveis os invés de produtos convencionais se houvesse subsídio para os produtos sustentáveis, e AMB3, "Nós estamos nos aproximando do limite sustentável da Terra"), os alunos do 8 semestre de Ciências Contábeis analisados são os que apresentaram as maiores médias (2,821, 3,786 e 4,143, respectivamente) e os alunos do 20 semestre do curso foram os que apresentaram as menores médias $(2,045,3,091$ e 3,048, respectivamente). É possível afirmar, assim, que os alunos da turma do 8o semestre participantes desta pesquisa são os que mais acreditam na melhor aceitação social para os consumidores de produtos sustentáveis, são os mais propensos a comprar produtos sustentáveis se estes produtos possuírem algum tipo de subsídio e são os mais preocupados com a limitação dos recursos disponíveis no planeta.

\section{CONSIDERAÇÕES FINAIS}

Este estudo foi desenvolvido com o objetivo de analisar o comportamento de consumo sustentável dos acadêmicos do curso de Ciências Contábeis da UFSM, tendo possibilitado identificar a postura desses alunos em relação à temática do consumo sustentável. Com base no modelo das 20 variáveis, proposto por Biswas e Roy (2015), pôde-se verificar que os estudantes entrevistados são do sexo feminino $(63,4 \%)$, o que reflete a atual realidade mundial da busca pela especialização do público feminino para competir no mercado de trabalho. Além disso, notou-se que maioria da amostra possui entre 21 e 24 anos (40\%), está no 5 o semestre (13,8\%) e frequenta o curso no turno da noite (56\%).

Os resultados descritos anteriormente permitiram uma análise detalhada de cada um dos cinco construtos pesquisados. Assim, pôde-se perceber que as seis variáveis que apresentaram maiores médias pertencem aos constructos valor condicional e valor ambiental, o que pode revelar que algumas condições são necessárias para que este público tenha um consumo mais consciente e, também, que estes estudantes já adquiriram certa consciência ambiental. Apesar de a pesquisa ter sido realizada com jovens acadêmicos, um público em que predomina a idade de até 25 anos e que está em uma fase específica de sua vida, estes podem ser influenciados por mudanças em sua situação, a qual influencia a sua decisão de compra (refletido pela alta média do valor condicional). Dessa forma, os resultados deste estudo corroboram a conclusão de Biswas e Roy (2015), que, embora tenha investigado os valores dos indivíduos de diferentes faixas etárias e nacionalidades que participaram de conferências na Índia, também apontou o valor condicional como o mais relevante. Pode-se entender, assim, que uma restrição orçamentária é capaz de influenciar a compra ou não 
de produtos verdes, sendo necessária uma análise de dados pertinentes à renda ou classe social dos acadêmicos para confirmar tal relação, o que não foi o foco desta pesquisa.

Outro aspecto relevante diz respeito à média mais baixa da variável SOC1, "Comprar produtos sustentáveis irá me ajudar a obter aceitação social", do constructo valor social, indicando que a aquisição de produtos sustentáveis não traz impacto positivo na relação com seu grupo social e que os acadêmicos parecem não ser suscetíveis às pressões sociais no que tange à mudança de comportamento que vem sendo demandada pela sociedade. Destaca-se, ainda, a baixa média da variável $\mathrm{CONH} 3$, "Busco informações a respeito dos insumos, processos e impactos relativos aos produtos antes de comprá-los", do constructo valor de conhecimento, que demonstra que os respondentes não costumam fazer uma análise mais aprofundada sobre a sustentabilidade do produto antes de adquiri-lo. A esse respeito, ressalta-se que o valor de conhecimento não foi apontado como um dos valores mais significativos pelos acadêmicos, mas foi o segundo valor mais importante observado por Biswas e Roy (2015). Assim, não se pode desconsiderar a existência de diferenças culturais entre Brasil e Índia, uma vez que isso influencia a forma como os indivíduos se relacionam com diferentes organizações em busca de informações acerca dos processos de fabricação e até mesmo dos próprios produtos que estão consumindo.

Ressalta-se que esta pesquisa possui limitações. Por tratar-se de uma amostra por conveniência, os resultados aqui apresentados referem-se aos estudantes que participaram desta pesquisa, impossibilitando sua inferência para os demais discentes de Ciências Contábeis da UFSM. Como sugestão para trabalhos futuros, pode-se destacar a necessidade de ampliar o público-alvo, contemplando outros cursos e outras instituições de ensino do município de Santa Maria, Rio Grande do Sul.

\section{REFERÊNCIAS}

BARBIERI, J. C.; SILVA, D. Desenvolvimento sustentável e educação ambiental: uma trajetória comum com muitos desafios. Revista de Administração Mackenzie, v. 12, n. 3, p. 5182, 2011.

BARTH, M.; RIECKMANN, M. Academic staff development as a catalyst for curriculum change towards education for sustainable development: an output perspective. Journal of Cleaner Production, p. 28-36, 2012.

BISWAS, A.; ROY, M. Green products: an exploratory study on the consumer behavior in emerging economies of the East. Journal of Cleaner Production, v. 87, p. 463-468, 2015.

CARS, M.; WEST, E.E. Education for sustainable society: attainments and good practices in Sweden during the United Nations Decade for Education for Sustainable Development (UNDESD). Journal of Environment,
Development and Sustainability, v. 17, p. 1-21, 2015.

CADASTRO GERAL DE EMPREGADOS E DESEMPREGADOS (CAGED). Cresce a participação da mulher no mercado de trabalho. Disponível em:<http://www.mte. gov.br/imprensa/cresce-a-participacao-damulher-no-mercado-de-trabalho.htm $>$. Acesso em: 25 jun. 2015.

COMISSÃO MUNDIAL SOBRE MEIO AMBIENTE E DESENVOLVIMENTO -CMMAD. Nosso futuro comum. Rio de Janeiro: Fundação Getúlio Vargas, 1991.

FURRIELA, R. B. Educação para o consumo sustentável. Ciclo de Palestras sobre Meio Ambiente Programa Conheça a Educação do Cibec/Inep-MEC/SEF/COEA, 2001. Disponível em: <http://download.inep.gov.br/download/ cibec/pce/2001/47-55.pdf>. Acesso em: 18 jun. 2015.

JACOBI, P. Educação ambiental, cidadania e 
sustentabilidade. Cadernos de Pesquisa, n. 118, p. 189-205, 2003.

JACOBI, P. R.; RAUFFLET, E.; ARRUDA, M. P. Educação para a sustentabilidade nos cursos de administração: reflexão sobre paradigmas e práticas. Revista de Administração Mackenzie, v. 12, n. 3, 2011.

KOLLMUSS, A.; AGYEMAN, J. Mind the gap: why do people act environmentally and what are the barriers to pro-environmental behavior? Environmental Education Research, n. 8. p. 239-260, 2002.

LAROCHE, M.; BERGERON, J.; BARBAROFORLEO, G. Targeting consumers who are willing to pay more for environmentally friendly products. Journal of Consumer Marketing, v.18, n. 6, p. 503-521, 2001.

LIN, P. C.; HUANG, Y. H. The influence factors on choice behavior regarding green products based on the theory of consumption values. Journal of Cleaner Production, n. 22, p. 11-18, 2012.

MARJAINÉ SZERÉNYI Zs; ZSÓKA, A.; SZÉCHY A. Consumer behavior and lifestyle patterns of Hungarian students with regard to environmental awareness. Society and Economy, n.33, p. 89-110, 2011.

MICHAUD, C.; LLERENA, D.. Green Consumer Behaviour: an Experimental Analysis of Willingness to Pay for Remanufactured Products. Business Strategy and the Environment, v.20, p. 408-420, 2011.

MINISTÉRIO DO MEIO AMBIENTE (MMA). Responsabilidade socioambiental. Disponível em: http://www.ministeriodomeioambiente. gov.br/responsabilidade-socioambiental. Acesso em: 15 maio 2015.

PEATTIE, K. Green consumption: behavior and norms. The Annual Review of Environment and Resources, v. 35, p. 195-228, 2010.

PINSONNEAULT, A.; KRAEMER, K. L. Survey research in management informations systems: an assessement. Journal of Management Information Systems, v.10, n. 2, p. 75-106, 1993.

POLONSKY, M. J.; ROSENBERGER III, P. J. Reevaluating green marketing: a strategic approach. Business Horizons, v. 44, n. 5, p. 2130, 2001.

RELAÇÃO ANUAL DE INFORMAÇÕES SOCIAIS DO MINISTÉRIO DO TRABALHO E EMPREGO (RAIS). Disponível em:<http://www.mte. gov.br/imprensa-mercado-de-trabalho-raiscrescimento-mulheres.htm>. Acesso em: 25 jun. 2015.

RA'NAEE, H.; KORDSHULI, Y.; BUZANJANI, A. An analysis of effects of marketing on consumer's decision to purchase green products (Case Study: the customers of Pegah's Dairy Production Group in Shiraz Town). Quarterly Journal of Modern Marketing Studies. v. 11, n. 1, p.165-171, 2012.

REX, E.; BAUMANN, H. Beyond ecolabels: what green marketing can learn from conventional marketing. Journal of Cleaner Production, v. 15, p. 567-576, 2007.

RICHARDSON, R. J. Pesquisa social: métodos e técnicas. São Paulo: Atlas, 1999.

ROBERTS, J.A. Green consumers in the 1990s: profile and implications for advertising. Journal of Business Research, v. 36, n. 3, p.217-231, 1996.

SILVA, M. E. et al .Um espelho, um reflexo! A Educação para a sustentabilidade como subsídio para uma tomada de decisão consciente do administrador. Revista de Administração Mackenzie, v. 14, n. 3, p. 154182, maio/jun.2013.

SILVA, M. E.; GÓMEZ, C. R. P. Consumo consciente: o papel contributivo da educação. Revista Reuna, v. 15, n. 3, p. 43-54, 2010.

TRIVINOS, A. N. S. Introdução a pesquisa em 
ciências sociais: a pesquisa qualitativa em educação. São Paulo: Atlas, 1987.

WANG, P.; QIAN, L.; YU, Q. Factors influencing sustainable consumption behaviors: a study of rural residents in China. Journal of Cleaner Production, p. 1-14, 2013.

ZSOKA, A. et al. Greening due to environmental education? Environmental knowledge, attitudes, consumer behavior and everyday pro-environmental activities of Hungarian high school and university students. Journal of Cleaner Production, v. 48, p. 126-138, 2013. 